\title{
Generalized two-dimensional Yang-Mills theory is a matrix string theory
}

\author{
M. Billò, M. Caselle, A. D’Adda ${ }^{\mathrm{a}}$ and P. Provero ${ }^{\mathrm{b}}$ \\ a Dipartimento di Fisica Teorica \\ Università di Torino \\ Via P. Giuria 1, I-10125 Torino, Italy \\ bDipartimento di Scienze e Tecnologie Avanzate \\ Università del Piemonte Orientale \\ I-15100 Alessandria, Italy
}

\begin{abstract}
We consider two-dimensional Yang-Mills theories on arbitrary Riemann surfaces. We introduce a generalized Yang-Mills action, which coincides with the ordinary one on flat surfaces but differs from it in its coupling to two-dimensional gravity. The quantization of this theory in the unitary gauge can be consistently performed taking into account all the topological sectors arising from the gauge-fixing procedure. The resulting theory is naturally interpreted as a Matrix String Theory, that is as a theory of covering maps from a two-dimensional world-sheet to the target Riemann surface.
\end{abstract}

Report-no DFTT 2/2000. Presented by A. D'adda at the Third Meeting on Constrained Dynamics and Quantum Gravity, Villasimius (Sardinia, Italy) September 13-17, 1999; to appear in the proceedings.

\section{INTRODUCTION}

In the early seventies 't Hooft [1] established that in the limit of a large number $N$ of colours gauge theories are dominated by planar diagrams, namely by Feynman diagrams with the topology of a sphere, and that diagrams of genus $g$ are suppressed by a factor $\left[\frac{1}{N}\right]^{2 g-2}$. The expansion in powers of $\frac{1}{N}$ can thus be reinterpreted as a perturbative string expansion, with string coupling constant $\frac{1}{N}$. In two space-time dimensions, gauge fields have no physical degrees of freedom and pure Yang-Mills theory (YM2) is invariant under area preserving diffeomorphisms, thus revealing its almost topological nature. As a consequence of these features it can be exactly solved on arbitrary Riemann surfaces, both in a lattice 2,3] and continuum [4 60rmulation. The interpretation in terms of a string theory of this solution was provided in a series of beautiful papers [7, 8] where it was shown that the coefficients of the $\frac{1}{N}$ expansion of the YM2 partition function count the number of maps (without foldings) from a two-dimensional world-sheet of genus- $g$ to the
Euclidean space-time, which is a Riemann surface of genus $G$. In two dimensions the number of maps is the number of string configurations and, as each map is weighed by the area of its world sheet, one can conclude that in this limit YM2 is a two-dimensional string theory.

A string theory can be associated to a (generalized) YM2 theory in a completely different way, inspired by Matrix String Theory (MST) 9, 10]. This new approach, introduced in Refs. 11.12 for the case of the cylinder and the torus, and generalized in 13 to arbitrary Riemann surfaces, will be the subject of this talk. It originates quite naturally from quantization of YM2 in a non-propagating gauge, the unitary gauge, but it requires a generalization of YM2 in the sense of 14, 15. On the other hand, the string interpretation is not related in this case to the expansion in powers of $\frac{1}{N}$, and it is valid for any value of $N$.

The plan of this contribution is the following. In Sec. 2 we review the exact solution of lattice YM2, and its interpretation as a string theory in the large- $N$ limit. Sec. 3 contains a general discussion of MST; Sec. 4 discusses the quantization 
of YM2 in the unitary gauge. In Sec. 5 we introduce the generalized YM2 and its interpretation as a MST. In Sec. 6 we extend the previous considerations to Riemann surfaces with boundaries, while in Sec. 7 we introduce a lattice gauge theory whose partition function coincides with the one of our generalized YM2. Sec. 8 is devoted to some concluding remarks and outlines of future research.

\section{YM2 ON ARBITRARY RIEMANN SURFACES}

The exact expression of the partition function of YM2 on a general Riemann surface was first obtained on the lattice by Rusakov [3], based on previous work by Migdal [2]. It is convenient to consider the lattice theory defined by the heatkernel action: if the product of the group elements around a plaquette is $V$, its Boltzmann weight is

$\mathrm{e}^{-S_{t}(V)}=\sum_{R} d_{R} \chi_{R}(V) \mathrm{e}^{-\frac{t}{2} C_{R}}$,

where the sum is over all the irreducible representations $R$ of the gauge group, $d_{R}$ is the dimensionality and $C_{R}$ the quadratic Casimir of the representation, $\chi_{R}(V)$ the character of $V$, and $t=g_{\mathrm{YM}}^{2} A$, with $g_{\mathrm{YM}}$ the gauge coupling and $A$ the area of the plaquette. Notice that $t$ is adimensional and it measures the area in units of the dimensional coupling constant $g_{\mathrm{YM}}$.

This action has a unique property: if we integrate over a link variable $U$ belonging to two adjoining plaquettes $A_{1}$ and $A_{2}$ we obtain exactly the Boltzmann weight corresponding to the heat kernel action for the resulting plaquette $A_{1} \cup A_{2}$. In formulae this reads:

$$
\int d U \mathrm{e}^{-S_{t_{1}}\left(V_{1} U^{\dagger}\right)-S_{t_{2}}\left(U V_{2}\right)}=\mathrm{e}^{S_{t_{1}+t_{2}}\left(V_{1} V_{2}\right)} .
$$

This property is a simple consequence of the orthogonality of the characters, and it allows to reduce, by successive integrations, any kernel with an arbitrary number of boundaries to a single plaquette with the sides suitably identified, and eventually to compute it exactly by using again well known properties of the characters. On the other hand, the continuum limit can be reached smoothly by adding more and more links to make the lattice spacing smaller and smaller, and the same renormalization invariance (2) insures that the result obtained on the lattice coincides with the one in the continuum.

The result of the calculation for a general Riemann surface of genus $G$ with an arbitrary number $p$ of boundaries is

$$
\begin{aligned}
& K_{G, p}\left(g_{1}, \ldots, g_{p} ; t\right) \\
& =\sum_{R} d_{R}^{2-2 G-p} \chi_{R}\left(g_{1}\right) \ldots \chi_{R}\left(g_{p}\right) \mathrm{e}^{-\frac{t}{2} C_{R}},
\end{aligned}
$$

where the $g_{i}$ 's are the group elements defined on the boundaries.

For $p=0$ we obtain the partition function

$Z_{G}(t)=\sum_{R} d_{R}^{2-2 G} \mathrm{e}^{-\frac{t}{2} C_{R}}$.

From now on we will consider the gauge group $\mathrm{U}(\mathrm{N})$ only, whose irreducible representations are labeled by $N$ integers

$n_{1}>n_{2}>\ldots>n_{N}$.

The corresponding dimensions $d_{R}$ and Casimir invariants $C_{R}$ are given by:

$$
\begin{aligned}
d_{R} & =\prod_{i>j}\left(n_{i}-n_{j}\right), \\
C_{R} & =\sum_{i} n_{i}^{2},
\end{aligned}
$$

so that the partition function reads

$$
\begin{aligned}
Z_{G}(N, t)= & \sum_{n_{1}>n_{2}>\ldots>n_{N}} \prod_{i>j}\left(n_{i}-n_{j}\right)^{2-2 G} \\
& \cdot \mathrm{e}^{-\frac{t}{2} \sum_{i} n_{i}^{2}} .
\end{aligned}
$$

In Refs. [7, 8] the large- $N$ limit of Eq. (8) was investigated. The limit was taken, as introduced by 't Hooft [1], by sending $N \rightarrow \infty$ and $t \rightarrow 0$ while keeping $\tilde{t} \equiv N t$ constant.

The result of the analysis is that the partition function (8) in such limit can be interpreted as the theory of a string living in the same two-dimensional target space $M_{G}$ as the original gauge theory. Each string configuration is described by a covering map from a two-dimensional 
world-sheet $W_{g}$ into $M_{G}$. The admissible string configurations have no folds, so that the area of the world-sheet is an integer multiple of the area of the target manifold. The covering maps are allowed to have branch points.

The equivalence of YM2 with the string theory described above is expressed by the following equation!:

$Z_{G}(N, t) \underset{N \rightarrow \infty}{\sim} \sum_{i, g, n} N^{2-2 g} \mathrm{e}^{-n \tilde{t} / 2}(\tilde{t})^{i} \omega_{g, G}^{n, i}$.

In (9), $\omega_{g, G}^{n, i}$ is the number of $n$-coverings of the target manifold with genus $g$ and $i$ branch points.

\section{MATRIX STRING THEORIES (MST)}

The interpretation of a generalized YM2 as a string theory that we want to present is inspired to the mechanism through which string states emerge in MST. We begin by briefly introducing a definition of Matrix String Theories sufficiently general to encompass both the original work [10] and our description of YM2. Consider a gauge theory defined on a Riemann surface, and containing at least one field $F$ transforming in the adjoint representation of the gauge group: in the case of $\mathrm{U}(\mathrm{N}), F$ is a hermitean $N \times N$ matrix. A possible gauge choice consists in diagonalizing $F$, that is in conjugating it into an element of the Cartan sub-algebra.

This gauge choice is characterized by a Gribov ambiguity related to the Weyl group, namely the group of invariance of the Cartan sub-algebra, which in our case is the symmetric group $S_{N}$ : in fact given a hermitian matrix $F$ there exist $N$ ! matrices that diagonalize $F$, corresponding to all possible permutations of the eigenvalues.

So in order to fix the gauge completely one has to choose in each point one of the $N$ ! Gribov copies. It is easy to see that in general this cannot be done smoothly on the whole Riemann surface whenever the first homotopy group of the surface is non-trivial.

\footnotetext{
${ }^{1}$ We consider here a simplified case where the sum over representations is restricted to "chiral" representations of $\mathrm{U}(\mathrm{N})$. Also the so called $\Omega$-point type of singularities, which are present for $G>1$, have been neglected. See \& for details.
}

In fact let $\gamma$ be a homotopically non-trivial closed path based in a point $x$, and choose one of the Gribov copies in $x$, say the one, named standard in the following, where $F=\operatorname{diag}\left(\lambda_{i}\right)$ with $\lambda_{1}(x) \geq \lambda_{2}(x) \geq \ldots \geq \lambda_{N}(x)$. If we diagonalize $F$ smoothly along $\gamma$ and we return to $x$, we will in general end up on a different Gribov copy, related to the standard one by a permutation $P(\gamma)$.

In this way we can associate a permutation to each homotopy class of closed paths based in $x$. It is easy to convince oneself that this defines a group homomorphism

$P: \pi_{1}(M ; x) \rightarrow S_{N}$.

We conclude that each configuration of the adjoint field $F$ belongs to a topological sector identified by a group homomorphism (10).

Consider for example the case of the torus: the homotopy group is generated by two cycles $a$ and $b$ with the relation

$a b=b a$.

Therefore to each configuration of the field $F$ and to each base point $x$ we can associate a topological sector defined by a pair of commuting permutations $\left(P_{1}, P_{2}\right)$; changing the base point $x$ results in changing the pair $\left(P_{1}, P_{2}\right)$ into a conjugate pair $\left(Q P_{1} Q^{-1}, Q P_{2} Q^{-1}\right)$.

The homomorphisms (10) are in one-to-one correspondence with the $\mathrm{N}$-coverings of the surface. This correspondence can be easily visualized by imagining each eigenvalue of $F$ as living on one of the $N$ sheets of the covering. Therefore we seem to be in a position to conclude that for a general Riemann surface, any theory with a U(N) gauge symmetry and at least one field transforming in the adjoint representation can be written as a sum over coverings of the surface, that is as a string theory.

While this is true in principle, it is of real interest only if the resulting string theory is free or weakly coupled, that is if the different sheets of the $N$-covering (i.e. the different eigenvalues of $F$ ) do not interact among themselves or interact weakly.

In general, however, strong interactions between the eigenvalues of $F$ do occur, coming from two different sources: 
1. from the functional integration over the other fields of the theory;

2. from the Faddeev-Popov determinant induced by the gauge-fixing.

The latter source of interactions is obviously always present, since the Faddeev-Popov determinant is just the Vandermonde determinant of the eigenvalues:

$\Delta_{\mathrm{FP}}=\exp \int d^{2} x 2 \sqrt{g} \sum_{i>j} \log \left|\lambda_{i}-\lambda_{j}\right|$.

Therefore, for the gauge theory to be a (weakly coupled) string theory the Vandermonde determinant must be canceled by the integration over other fields.

In Ref. [9, 10], a MST was introduced as a nonperturbative formulation of type-IIA superstring theory. It is described in terms of a supersymmetric, two-dimensional $\mathrm{U}(\mathrm{N})$ gauge theory with action

$$
\begin{aligned}
S & =\int d^{2} \xi \operatorname{Tr}\left[D_{\mu} X^{i} D^{\mu} X^{i}+\frac{1}{2} g_{\mathrm{s}}^{2} F_{\mu \nu} F^{\mu \nu}\right. \\
& +\frac{1}{2 g_{\mathrm{s}}^{2}}\left[X^{i}, X^{j}\right]^{2}-i \bar{\theta} \gamma^{\mu} D_{\mu} \theta \\
& \left.-\frac{1}{g_{\mathrm{s}}} \theta^{T} \Gamma_{i}\left[X^{i}, \theta\right]\right]
\end{aligned}
$$

where $g_{\mathrm{s}}$ is the string coupling, $X^{i}(i=1, \ldots, 8)$ are $N \times N$ hermitean matrices representing the transverse space-time coordinates, $\theta$ their fermionic superpartners, and $F$ is the $\mathrm{U}(\mathrm{N})$ field strength.

In the limit $g_{\mathrm{s}} \rightarrow 0$ all the fields $X$ and $\theta$ commute and can be diagonalized simultaneously. This diagonalization produces the topological sectors as described above. The cancellation of the Vandermonde determinant is achieved through supersymmetry: the contribution of the fermionic fields $\theta$ cancels the one of the $X$ fields, so that the strong interaction between the eigenvalues disappears and the theory indeed describes free strings propagating in 8 transverse dimensions. For nonzero $g_{\mathrm{s}}$, weak string interactions of order $g_{\mathrm{s}}^{2}$ are generated by the non-diagonal terms.

\section{YM2 IN THE UNITARY GAUGE}

In this section we will discuss the quantization of YM2 in the unitary gauge, namely in the gauge where the field strength $F$ is diagonal. This condition does not fix the gauge completely as it leaves a $\mathrm{U}(1)^{N}$ gauge invariance, so that further gauge fixing conditions for the abelian part have to be introduced separately. The unitary gauge is a non-propagating gauge (the non-diagonal components of the ghost-antighost fields do not propagate) and in four dimensions it makes the theory non-manifestly renormalizable. As we shall see, also in two dimensions this gauge is plagued by divergences that occur when two eigenvalues coincide. However, these can be eliminated by considering a suitable generalized YM2 (in the sense of [14,15). This procedure also eliminates the interaction between different eigenvalues induced by the Vandermonde determinants, thus ensuring that the strings associated to the topological sectors discussed in the previous section are essentially free.

The starting point is the first order formalism for YM2 on a genus- $G$ Riemann surface: the partition function is

$$
\begin{aligned}
Z & =\int[d A][d F] \exp \left[-\int_{\Sigma_{G}} d \mu V(F)\right. \\
& \left.+\mathrm{i} \operatorname{Tr} \int f(A) F\right]
\end{aligned}
$$

where

$f(A)=d A+A \wedge A$,

$\mathrm{F}$ is an auxiliary field transforming in the adjoint representation of $\mathrm{U}(\mathrm{N})$, and $V(F)$ is a gaugeinvariant potential, depending on the eigenvalues of $F$ only. This defines a generalized YangMills theory [14,15]; ordinary YM2 is recovered by choosing

$V(F)=\operatorname{Tr} \frac{t}{2} F^{2}$

and integrating over $F$ to obtain the usual secondorder formalism.

To determine whether such a theory is a MST, we have to

1. fix the gauge where $F$ is diagonal; 
2. check whether the Vandermonde determinant coming from the gauge fixing can be canceled by terms coming from other fields.

The BRST-invariant action for the unitary gauge is of the form

$S_{\mathrm{BRST}}=S_{\text {Cartan }}+S_{\text {off-diag }}$,

where $S_{\text {Cartan }}$ depends only on the eigenvalues of $F$ and the diagonal components of the $A$ fields:

$S_{\text {Cartan }}=\int_{\Sigma_{G}}\left[V(\lambda)-\mathrm{i} \sum_{i=1}^{N} \lambda_{i} d A^{(i)},\right]$

while $S_{\text {off-diag }}$ is given by

$$
\begin{aligned}
S_{\text {off-diag }} & =\int d \mu \sum_{i>j}\left(\lambda_{i}-\lambda_{j}\right)\left[\frac{1}{2} \epsilon^{a b} \hat{A}_{a}^{i j} \hat{A}_{b}^{j i}\right. \\
& \left.+\mathrm{i} c^{i j} \bar{c}^{j i}+\mathrm{i} c^{j i} \bar{c}^{i j}\right]
\end{aligned}
$$

where

$\hat{A}_{a}^{i j} \equiv E_{a}^{\mu} A_{\mu}^{i j}$,

with $E_{a}^{\mu}$ the inverse vierbein; $c$ and $\bar{c}$ are the ghost and anti-ghost fields. If YM2 is a MST, the integrations over $\hat{A}$ and $c, \bar{c}$ must cancel each other.

Note that at the classical level $S_{\text {off-diag }}$ is invariant under the following transformation, depending on four Grassmann-valued parameters $\eta$, $\zeta, \xi, \chi$ :

$$
\begin{aligned}
\delta \hat{A}_{0} & =\mathrm{i}(\eta c+\zeta \bar{c}), \\
\delta \hat{A}_{1} & =\mathrm{i}(\xi c+\chi \bar{c}), \\
\delta c & =-\chi \hat{A}_{0}+\zeta \hat{A}_{1}, \\
\delta \bar{c} & =-\xi \hat{A}_{0}+\eta \hat{A}_{1} .
\end{aligned}
$$

This "supersymmetry" does indeed seem to guarantee the cancellation of the contribution of $S_{\text {off-diag. }}$

However, it is known 16] that this supersymmetry is in general broken by quantum effects, the anomaly being proportional to the scalar curvature of $\Sigma_{G}$ :

$$
\begin{aligned}
& \int[d c][d \bar{c}][d A] \mathrm{e}^{-S_{\text {off }- \text { diag }}} \\
& =\exp \frac{1}{8 \pi} \int_{\Sigma_{G}} d \mu R \sum_{i>j} \log \left|\lambda_{i}-\lambda_{j}\right| .
\end{aligned}
$$

This anomaly is essentially due to the fact that for a curved surface (twice) the number of 0 -forms (the $c$ and $\bar{c}$ fields) does not equal the number of 1-forms (the field $A$ ).

Inserting this result into the partition function we obtain an effective theory for the diagonal degrees of freedom that exhibit a residual $\mathrm{U}(1)^{\mathrm{N}}$ gauge invariance:

$$
\begin{aligned}
Z & =\int\left[d A^{(i)}\right]\left[d \lambda_{i}\right] \exp -\int_{\Sigma_{G}}[\tilde{V}(\lambda) d \mu \\
& \left.-\mathrm{i} \sum_{i} \lambda_{i} d A^{(i)}\right]
\end{aligned}
$$

with

$\tilde{V}(\lambda)=V(\lambda)-\frac{1}{8 \pi} \int_{\Sigma_{G}} R \sum_{i>j} \log \left|\lambda_{i}-\lambda_{j}\right|$.

The last term at the r.h.s. of (27) is singular when two eigenvalues coincide and the problem of a consistent regularization leading to the standard expression (8) for the partition function is essentially unsolved.

According to Ref. 16, the procedure to recover (8) from (26) involves two steps:

1. The $\mathrm{U}(1)^{N}$ gauge fixing and the functional integration over the $A^{(i)}$ fields are performed, and the result is equivalent to replacing all the eigenvalues $\lambda_{i}(x)$ with integer-valued constants $n_{i}$ (the flux of the abelian field strength) and summing over the $n_{i}$ 's. One obtains

$$
Z=\sum_{n_{1}, \ldots, n_{N}} \prod_{i>j}\left(n_{i}-n_{j}\right)^{2-2 G} \mathrm{e}^{-V(n)} .
$$

2. The terms with two integers $n_{i}$ coinciding, to be referred to in the following as nonregular terms, are divergent and are essentially discarded by hand.

In this way, with $V(n)=\frac{t}{2} \sum_{i} n_{i}^{2}$ one recovers Eq. (8).

Discarding non-regular terms also means discarding the non-trivial topological sectors described in Sec. 3. In fact in a non-trivial topological sector there is at least one homotopically non-trivial closed path that induces a non-trivial 
permutation on the eigenvalues. As the eigenvalues are constant integers, this implies that at least two eigenvalues coincide. In the following section we propose an alternative approach, that allows to eliminate all divergences while keeping all nontrivial sectors at the price, however, of modifying the coupling of YM2 to gravity.

\section{GENERALIZED YM2 IS A MATRIX STRING THEORY}

The divergences of the theory in the unitary gauge can be eliminated, rather than by the ad hoc procedure of Ref. 16., by a redefinition of the theory, namely by using the arbitrariness of the potential $V(\lambda)$ to absorb the divergent term due to the anomaly. In other words since all choices of $V(\lambda)$ define a legitimate generalized Yang-Mills theory, it is apparent that there is a specific choice in which the anomaly cancels and one obtains a Matrix String Theory: namely we can choose $V(\lambda)$ in such a way that the anomaly term cancels and $\tilde{V}(\lambda)$ is regular when two eigenvalues coincide.

For example we can choose $V(\lambda)$ such that

$\tilde{V}(\lambda)=\frac{t}{2} \sum_{i} \lambda_{i}^{2}$.

With this choice we define a theory whose action coincides with the one of ordinary YM2 on flat surfaces, but differs from it when coupled to twodimensional gravity (recall that the anomaly (25) is proportional to the curvature of the Riemann surface).

The partition function of the generalized YM2 defined by the potential (29) consists of topological sectors which are in one-to-one correspondence with the inequivalent coverings of the target manifold. There is no interaction among different eigenvalues, so the partition function describes a theory of non interacting strings. It is clear from Eq. 26) that a U(1) gauge theory is defined on each connected component of the covering, that is of the world sheet. The product of the partition functions of these U(1) gauge theories gives the Boltzmann weight of the covering. Although a compact expression for the partition function is in general not available we present here the recipe for writing it down for a general Riemann surface without boundaries (the case of a surface with boundaries will be discussed in Sec. 6 ). It is convenient to work directly on the generating function of the partition functions for arbitrary $N$, namely the grand-canonical partition function. This is defined as

$Z(G, q)=\sum_{N=0}^{\infty} Z_{N}(G) q^{N}$

where $Z_{N}(G)$ is the $\mathrm{U}(\mathrm{N})$ partition function. For a given Riemann surface $\Sigma_{G}$ of genus $G$ one proceeds as follows:

1. Write the grand-canonical partition function of unbranched coverings of $\Sigma_{G}$ :

$$
Z^{(\mathrm{cov})}(G, q)=\sum_{k=0}^{\infty} Z_{k}^{(\mathrm{cov})}(G) q^{k}
$$

where $Z_{k}^{(\operatorname{cov})}(G)$ is the number of inequivalent $k$-coverings of $\Sigma_{G} \cdot Z^{(\text {cov })}(G, q)$ has been studied in the literature [8, 17, 18] and is known in closed form.

2. Take the logarithm of $Z^{(\operatorname{cov})}(G, q)$ to obtain the free energy

$$
\begin{aligned}
F^{(\mathrm{cov})}(G, q) & =\log Z^{(\mathrm{cov})}(G, q) \\
& =\sum_{k=0}^{\infty} F_{k}^{(\operatorname{cov})}(G) q^{k}
\end{aligned}
$$

where $F_{k}^{(\operatorname{cov})}(G)$ is the number of connected $k$-coverings of $\Sigma_{G}$. No closed expression is known for $F^{(\operatorname{cov})}(G, q)$.

3. Associate to each connected covering the partition function of the U(1) gauge theory living on it, to obtain the free energy of the generalized YM2:

$$
F(G, q, t)=\sum_{k=0}^{\infty} F_{k}^{(\operatorname{cov})}(G) Z_{\mathrm{U}(1)}(k, t) q^{k}
$$

Here $Z_{\mathrm{U}(1)}(k, t)$ is the partition function of a (generalized) U(1) gauge theory on a Riemann surface of area $k t$ (in units of 
the gauge coupling constant). $Z_{\mathrm{U}(1)}(k, t)$ does not depend on the genus and for the quadratic potential (29) it reads]:

$Z_{\mathrm{U}(1)}(k, t)=\sum_{n=-\infty}^{\infty} \mathrm{e}^{-\frac{1}{2} k t n^{2}}$

Therefore

$F(G, q, t)=\sum_{n=-\infty}^{\infty} F^{(\text {cov })}\left(G, \mathrm{e}^{-\frac{1}{2} t n^{2}} q\right)$

4. The gauge theory partition function is then

$$
\begin{aligned}
Z(G, q, t) & =\mathrm{e}^{F(G, q, t)}=\sum_{N=0}^{\infty} Z_{N}(t, G) q^{N} \\
& =\prod_{n} Z^{(\mathrm{cov})}\left(G, \mathrm{e}^{-\frac{1}{2} t n^{2}} q\right) \cdot(36)
\end{aligned}
$$

As an example, we will treat explicitly the case of the torus. This is the only case (except for the trivial one of the sphere) where a closed expression is available for the number of connected coverings. As discussed in Sec. 3, the coverings of the torus are in one-to-one correspondence with the pairs of commuting permutations. The number of such pairs is $N ! p(N)$ where $p(N)$ is the number of partitions of $N$. Therefore there are $p(N)$ inequivalent coverings (that is, not counting as different two coverings that can be obtained from each other by simple relabeling of the sheets) and we have:

$$
\begin{aligned}
Z^{(\mathrm{cov})}(G=1, q) & =\sum_{N=0}^{\infty} p(N) q^{N} \\
& =\prod_{k=1}^{\infty} \frac{1}{1-q^{k}}
\end{aligned}
$$

For the connected coverings we have

$F^{(\mathrm{cov})}(G=1, q)=\sum_{N=1}^{\infty} \sum_{r \mid N} \frac{1}{r} q^{N}$

where the second sum is extended to all divisors of $N$ (including $N$ ).

\footnotetext{
${ }^{2}$ The generalization to arbitrary potentials is straightforward
}

Therefore we obtain for the YM2 free energy the expression

$$
F(G=1, q, t)=\sum_{n=-\infty}^{\infty} \sum_{N=1}^{\infty} \sum_{r \mid N} \frac{1}{r} \mathrm{e}^{-\frac{1}{2} N t n^{2}} q^{N}
$$

and for the partition function

$$
Z(G=1, q, t)=\prod_{n=-\infty}^{\infty} \prod_{k=1}^{\infty} \frac{1}{1-q^{k} \mathrm{e}^{-\frac{1}{2} k t n^{2}}} .
$$

This result does not coincide with the expression (28) of the partition function for $G=1$. To recover the "usual" partition function on the torus, one has to consider only the topological sectors corresponding to pairs of commuting permutations of the special form $(1, P)$, weighted with a factor $(-1)^{n_{c}}$ where $n_{c}$ is the number of connected components of the covering. One can check that this prescription effectively cancels all non-regular terms from Eq. (28).

Therefore for the case of the torus, where the Riemann curvature vanishes and our generalized YM2 coincides with the usual one at the classical level, the inclusion of all the topological sectors leads to a different quantum theory which is richer in structure and closely related to the MST of Ref. [10], as shown in Refs. [19,20].

\section{SURFACES WITH BOUNDARIES}

Consider now a Riemann surface $\Sigma_{G, p}$ of genus $G$ with $p$ boundaries. The arguments of the preceding sections hold essentially unaltered, and the generalized YM2 can be written as a theory of $N$ coverings of $\Sigma_{G, p}$ with a U(1) gauge theory living on each connected component of the covering.

The natural quantity to be studied is the kernel that will depend on $p$ states defined on each boundary. For any given boundary, a state is completely specified by assigning:

1. A conjugacy class of permutations, defined as the conjugacy class of the permutation $P(\gamma)$ where $\gamma$ is any closed curve that can be continuously deformed into the boundary. It is given in terms of $N$ integers $r_{l}(l=$ $1, \ldots, N)$ satisfying $\sum_{l} l r_{l}=N$ where $r_{l}$ is the number of cycles of length $l$ in $P(\gamma)$. 
2. A U(1) holonomy associated to each cycle of the conjugacy class defined above.

The procedure to construct the kernels follows closely the one described above for surfaces without boundaries: one constructs the free energy of the coverings of $\Sigma_{G, p}$ and associates to each connected $k$-covering a $\mathrm{U}(1)$ kernel

$K_{\mathrm{U}(1)}(k t, \phi)=\sum_{n=-\infty}^{\infty} \mathrm{e}^{-\frac{1}{2} k t n^{2}+\mathrm{i} n \phi}$.

Exponentiation then produces the generalized YM2 kernel. Details can be found in Ref. 13. One must check the consistency of the theory, namely that the set of kernels constructed in this way produces consistent results when the surfaces are cut and sewn to produce new ones. This property is obviously true for the pure theory of coverings and it can be proved that the introduction of the $\mathrm{U}(1)$ gauge theory on each connected world sheet does not spoil it.

\section{LATTICE EFFECTIVE THEORY}

We have seen in the previous sections that, after integration over the non-diagonal components of the fields, our generalized YM2 theory is described by an effective theory of coverings with a $\mathrm{U}(1)$ gauge theory on each connected world sheet. In this section we show that this effective theory is equivalent to a lattice theory whose gauge group is the semi-direct product of $S_{N}$ and $\mathrm{U}(1)^{N}$. Consider first a surface with the topology of a disc. Since its fundamental group is trivial, the only $\mathrm{N}$ covering consists of $N$ disjoint copies of the disc: all closed paths are mapped into the identity by the homomorphism (10). From the discussion of the previous section it follows that the state on the boundary is given in terms of $N \mathrm{U}(1)$ invariant angles $\phi_{i}$. The kernel is then

$K((P, \phi), t)=\delta(P) \sum_{n_{i}} \mathrm{e}^{\sum_{i=1}^{N}\left(\mathrm{in}_{i} \phi_{i}-t v\left(n_{i}\right)\right)}$.

Consider now the subgroup $\mathcal{G}_{N}$ of $\mathrm{U}(\mathrm{N})$ given by the matrices of the form

$(P, \phi)=\operatorname{diag}\left(\mathrm{e}^{\mathrm{i} \phi_{1}}, \ldots, \mathrm{e}^{\mathrm{i} \phi_{N}}\right) P$, where the $\phi_{i}$ 's are real and $P$ is a permutation matrix:

$P_{i j}=\delta_{i P(j)}$.

The group product reads

$(P, \phi)(Q, \theta)=(P Q, \phi+P \theta)$,

which shows that $\mathcal{G}_{N}$ is a semi-direct product of $S_{N}$ and $\mathrm{U}(1)^{N}$.

The kernel (42) is invariant under gauge transformations belonging to $\mathcal{G}_{N}$ :

$K\left((Q, \theta)(P, \phi)(Q, \theta)^{-1}, t\right)=K((P, \phi), t)$.

Moreover it shares with the heat-kernel action (1) of lattice gauge theory the invariance under renormalization group transformations:

$$
\begin{gathered}
\sum_{Q \in S_{N}} \int \prod_{i} \frac{d \theta_{i}}{2 \pi} K\left(\left(P_{1}, \phi_{1}\right)(Q, \theta), t_{1}\right) \\
\cdot K\left((Q, \theta)^{-1}\left(P_{2}, \phi_{2}\right), t_{2}\right) \\
=K\left(\left(P_{1}, \phi_{1}\right)\left(P_{2}, \phi_{2}\right), t_{1}+t_{2}\right) .
\end{gathered}
$$

The kernel (42) can thus be used as a heathkernel type of action to construct a lattice gauge theory for the group $\mathcal{G}_{\mathcal{N}}$, with partition function

$Z_{\text {latt }}=\prod_{\alpha} \sum_{P_{\alpha}} \int_{0}^{2 \pi} d \phi_{\alpha} \prod_{\mathrm{pl}} K\left(\left(P_{\mathrm{pl}}, \phi_{\mathrm{pl}}\right), t\right)$,

where $\alpha$ enumerates the links in the lattice, and the product of all the elements $\left(P_{\alpha}, \phi_{\alpha}\right)$ of $\mathcal{G}_{N}$ along a plaquette defines the plaquette variable $\left(P_{\mathrm{pl}}, \phi_{\mathrm{pl}}\right)$. In complete analogy with the solution of YM2 obtained in [3], one can solve exactly the theory by reducing the surface to a single plaquette with suitably identified links. As shown in [13], the resulting expression coincides with the one that is obtained, via the steps discussed in the previous Section, by including all the topological sectors in the MST corresponding to our generalized YM2 theory.

\section{CONCLUSIONS AND FURTHER DE- VELOPMENTS}

Quantization of YM2 in a non-propagating gauge, such as the unitary gauge, leads to nonmanifestly renormalizable divergences as well as 
to the appearance of a structure of topological sectors, associated to the $N$ coverings of the target manifold ; this strongly suggests the presence of an underlying string theory. In order to recover the standard partition function obtained with other gauge choices, the divergences have to be removed more or less by hand, thus suppressing the topological sectors even in the case of torus where they are not related to any divergence.

However we found that the divergences can be removed, and the topological sectors maintained, by considering a generalized theory whose action coincides with standard one for vanishing Riemann curvature. Indeed one can say that the generalized theory differs from the conventional one for a non-standard coupling with gravity. In this approach the string theory does not emerge from the large $N$ limit, but directly for any value of $N$ from the topological structure of the theory as in MST. It is not a chance that for the case of the torus our partition function is closely related to the one of the full MST.

These considerations suggest some lines along which future research might develop. Two of them seem the most natural ones:

1. The string theory considered so far is a theory of free strings. It is natural to introduce interactions by including branched coverings, that is the possibility for strings to split and join. A branch point can be seen as a boundary where all the U(1) invariant angles are set to zero. In this sense our treatment of surfaces with boundaries includes branch points as well. However, the most interesting case is the one in which a continuum distribution of branch points is introduced, as was done in Refs. 17, 18 for the pure theory of coverings.

2. Other non-propagating gauges would require a different tuning of the potential to eliminate the divergences and may lead to more general theories. For instance a gauge choice where, for $N=L M$, the field strength is block diagonal, with blocks of size $M \times M$, would be equivalent to a theory of $L$-coverings with a non abelian $U(M)$ gauge theory on the world sheet. This formulation would include both standard YM2 $(L=1)$ and our generalized theory $(L=N)$ as limiting cases. As a string theory emerges in both cases through completely different mechanisms, some investigation will be needed to ascertain whether a deeper relation exists between these two different formulations.

\section{REFERENCES}

1. G. 't Hooft, Nucl.Phys. B75 (1974) 461.

2. A.A. Migdal, Sov. Phys. JETP 42 (1975) 413.

3. B. Ye. Rusakov, Mod. Phys. Lett. A5 (1990) 693.

4. E. Witten, Comm. Math. Phys. 141 (1991) 153.

5. E. Witten, J. Geom. Phys. 9 (1992) 303 hepth/9204083).

6. M. Blau and G. Thompson, Int. J. Mod. Phys. A7 (1992) 3781.

7. D. J. Gross, Nucl. Phys. B400 (1993) 161 (hep-th/9212149).

8. D. J. Gross and W. Taylor, Nucl. Phys. B400 (1993) 395 (hep-th/9301068).

9. L. Motl, hep-th/9701025.

10. R. Dijkgraaf, E. Verlinde and H. Verlinde, Nucl. Phys. B500 (1997) 43 hepth/9703030).

11. M. Billó, M. Caselle, A. D'Adda and P. Provero, Nucl. Phys. B543 (1999) 141 hepth/9809095).

12. M. Billó, M. Caselle, A. D'Adda and P. Provero, Proceedings Corfú Conference, A. Ceresole et al., eds., Springer LNP 525 (1998) (hep-th/9901053).

13. M. Billó, A. D'Adda and P. Provero, hepth/9911249.

14. M. R. Douglas, K. Li and M. Staudacher, Nucl. Phys. B420 (1994) 118 hepth/9401062).

15. O. Ganor, J. Sonnenschein and S. Yankielowicz, Nucl. Phys. B434 (1995) 139 hepth/9407114).

16. M. Blau and G. Thompson, in Proc. 1993 Summer School in High Energy Physics and Cosmology, E. Gava et al., eds., World Scien- 
tific (1994) (hep-th/9310144).

17. I. K. Kostov and M. Staudacher, Phys.Lett. B394 (1997) 75 (hep-th/9611011).

18. I. K. Kostov, M. Staudacher and T. Wynter, Commun. Math. Phys. 191 (1998) 283 (hepth/9703189).

19. I. Kostov and P. Vanhove, Phys. Lett. B444 (1998) 196 (hep-th/9809130).

20. G. Grignani and G. W. Semenoff, hepth/9903246. 